from the drain. About the fourth or fifth day the general condition of the patients improved, and some foul discharge came away alongside, and not through, the drain; came, in fact, from the abdominal wall. In such cases the edges of the wound felt indurated and were tender, and, if stitches were taken out, foul sloughy material escaped. Anaerobic infection had been locked up in the abdominal wall, and had led to necrosis of fat, aponeurosis, and muscle. It was this that had accounted for the pronounced post-operative constitutional symptoms. Surely it must be that this added abdominal wall infection in some cases turns the scale between recovery and death.

Since adopting the plan of leaving the abdominal wound open, packing with gauze, in which is incorporated a small Carrel tube (Fig. 3), through which small quantities

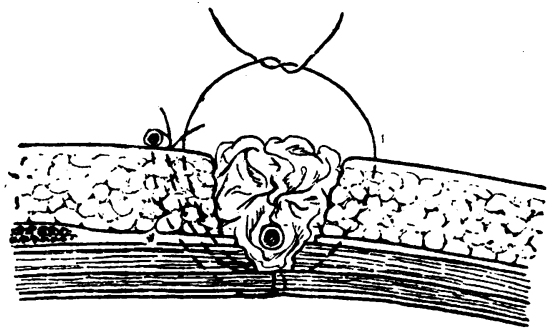

FIg. 3.-Open treatment of abdominal wound. Carrel tube secen on rection in deeper layers of gauze packing.

of hydrogen peroxide are injected every few hours, I have found that sloughing is obviated, constitutional symptoms are absent, and healing is not delayed. Postoperative hernia is also less common, as no muscle or aponcurosis is lost by necrosis. The avoidance of all catgut sutures, and the use of the silkworm-gut looped suture for peritoneum and muscle, leaves no dead organic material iff the wound as pabulum for bacteria (Fig. 4).

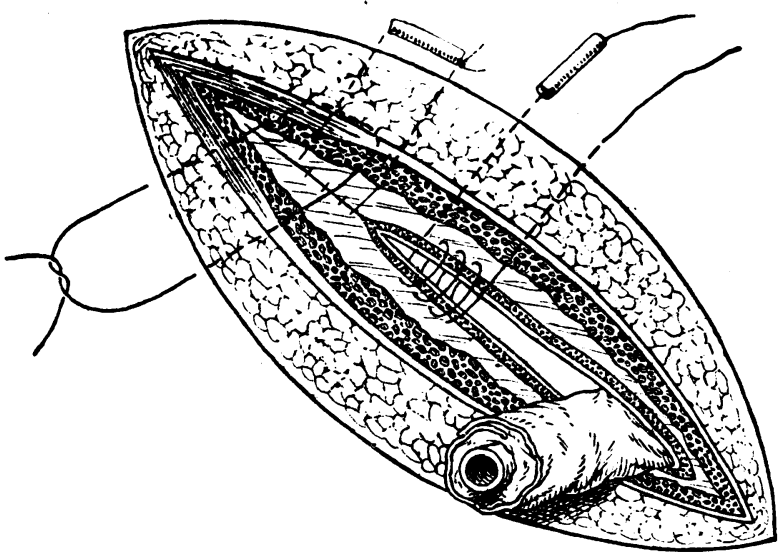

Fig. 4.-Method of using "looped sutures" of silkwormgut in place of catgut for closure of deeper layers of wound.

In stout subjects the open treatment of the abdominal wound is especially necessary, as anaerobic infection is tolerated badly.

\section{Post-operative Intestinal Obstruction}

This accounts for a number of deaths in patients who have successfully dealt with the peritoneal infection. Where the obstruction is organic in nature and due to a binding down of lower ileal coils to the appendix bed, the patient will complain of some peristaltic pains associated with his distension and vomiting. In such cases, following gastric lavage, a tube enterostomy, or an ileocolostomy, must be undertaken without delay, and the chloride loss from vomiting must be made good by subcutaneous saline infusions. Where, with distension, there is no clinieal evidence of ineffective peristalsis, and paresis

of the gut exists, continuous gastric drainage through a duodenal tube, the intravenous injection of $30 \mathrm{c} . \mathrm{cm}$. of 5 per cent. sodium chloride solution, to replace loss of chlorides and stimulate peristaltic contraction, the application of heat to the abdomen, and the use of pituitrin, may excite peristalsis. Enterostomy has a limited value in this type, but permits of feeding and the replacement of loss of fluid and salt.

\title{
The Value of Caecostomy
}

In a strictly limited number of cases, where at operation a grossly distended caecum is found, a valvular tube caecostomy is unquestionably indicated. The comfort which this gives, the ready supply of fluid, and the absence of flatulent distension, make this a valuable addition to an appendix operation in selected cases.

\section{Conclusions}

1. The mortality from " appendicitis" remains as high as it was twenty years ago, because, while facilities for treatment have improved, diagnosis in the fatal type of case has not.

2. There is now very strong evidence that the fatal cases of acute appendicular disease are primarily cases of obstruction and not inflammation of the appendix. It is necessary to distinguish between these two types of acute disease, the clinical symptoms and essential pathology of which differ widely. The obstructive cases must be regarded and treated as belonging to the same category of urgency as internal strangulation of the intestine.

3. Free exposure at operation is essential in the obstructive cases.

4. While immediate operation is expedient in the early stages of appendicitis, delay is advisable in the resolving case, the danger of operation being greater than that of expectancy.

5. Where a large localized abscess is present, simple drainage with minimal disturbance is the safest line of treatment.

6. In cases with perforation and peritonitis, infection of the abdominal wound may be an important factor in determining a fatal issue. Open treatment of the wound is therefore advisable.

7. Post-operative obstruction accounts for a number of deaths. A timely enterostomy or ileo-colostomy may prove a life-saving measure.

\section{THE FAMILIAL INFLUENCE IN GASTRIC FUNCTION}

BY

FRANK L. APPERLY, M.A., M.D., D.Sc. $\Lambda \mathrm{ND}$

JOAN H. NORRIS, M.Sc.

(From the Macleorl Research Laboratory, Pathology Department, University of Melbourne)

In the course of some recent investigations into the question of heredity in gastric cancer we had occasion to examine, by means of the fractional test meal, the chemical and motor functions of the stomach in several members of each of a number of families. During this investigation we were struck by the frequency with which members of the same family gave closely similar results. This has been commented upon by several writers, notably Hurst, ${ }^{1}$ who has on several occasions observed that duodenal ulcer with its accompanying "type" of stomach tends to run in families. The same has been noted with regard to achlorhydria and, to a less extent, "gastric diathesis." In 1923 one of us, " showed two instances of the close resemblance in the test-meal charts of parent and child. Since then we bave collected two 
more examples of the parent-and-child association, and, as shown in Group V, the similarity is seen in three out of these four cases.

\section{Criteria of the Diathesis}

In judging similarity of test-meal charts we have used three criteria-namely, emptying time for where this is more than $1 \frac{3}{4}$ hours, the volume of gastric contents recovered at that time), the shape of the curve, and the acidity. For purposes of classification we have divided these three criteria as follows:

1. Emptying Time.-(a) Rapid-stomach empty before 11 hours; (b) normal; (c) delayed-more than $30 \mathrm{c} . \mathrm{cm}$. found in the stomach at $1 \frac{3}{5}$ hours.

2. The Shape of the Curve.-(a) Climbing or steadily rising; (b) flat; (c) falling-that is, a rise in the curve followed by a definite fall.

3. Acidity.-(a) Hyperchlorhydria (free acidity over 45); (b) normal ; (c) hypochlorhydria (free acid present, but below 15) ; (d) achlorhydria.

In Table $\mathrm{A}$ are shown the gastric emptying times of healthy students, compiled-for comparison with the present series-from the combined figures of Bennett and Ryle," Baird, Campbell, and Hern," and Apperly and Semmens. ${ }^{5}$ Our divisions of the acidity values are based on Bennett and Ryle's graphs of acidity curves of healthy students.

TABLE A.-Rate of Emptying of the Gruel Meal (Figures given as percentages)

\begin{tabular}{|c|c|c|c|c|c|c|c|}
\hline \multirow{2}{*}{ Observers } & \multirow{2}{*}{$\begin{array}{c}\text { Average } \\
\text { Emptying } \\
\text { Time } \\
\text { (Hours) }\end{array}$} & \multicolumn{6}{|c|}{$\begin{array}{l}\text { Stomach contains no Starch at } \\
\text { (Hours) }\end{array}$} \\
\hline & & $0-1$ & $3 \frac{1}{2}$ & $1 \frac{1}{2}$ & 13 & 2 & $2+$ \\
\hline 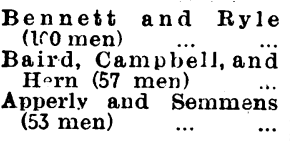 & $\begin{array}{l}21 \\
-\end{array}$ & $\begin{array}{l}5 \\
2\end{array}$ & $\begin{array}{l}7 \\
9\end{array}$ & $\begin{array}{l}14 \\
11\end{array}$ & $\begin{array}{r}9 \\
25\end{array}$ & $\begin{array}{l}17 \\
17\end{array}$ & $\begin{array}{l}48 \\
36\end{array}$ \\
\hline $\begin{array}{ccc}\text { Abcve groups combined } \\
(210 \mathrm{men}) & \ldots & \ldots\end{array}$ & - & 5 & 6 & 10 & 15 & 17 & 47 \\
\hline
\end{tabular}

In the present series two or more members of $\mathbf{3 1}$ families comprising 86 individuals are examined and divided into four groups according to whether all members exhibit similarity in all three criteria, or only two or one, or in none. In the fifth group are four parents and their children.

In Group I (comprising 10 families-29 individuals) there is close resemblance in the three criteria in all members examined of each family. These similarities are more obvious when the figures are plotted together on the same chart. In Group II ( 6 families-14 individuals) there is complete resemblance in the test-meal charts of all members of each family in two of the three criteria, or the majority of members exhibit complete similarity. In Group III (5 families-15 individuals) these resemblances extend to only one of our criteria, and in Group IV (6 families-20 individuals) to none.

While it is difficult or impossible to express these results completely by a graph, that shown below will suffice.

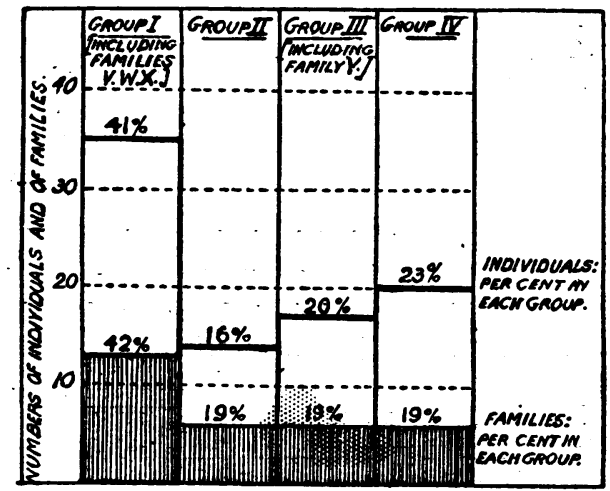

It will be seen that nearly half the number of all individuals examined (41 per cent.) belonged to families all members of which showed a complete resemblance to each other as regards their test-meal charts in all three criteria.

Group I.-Families in which the Test-meal Charts of all Members examined are Similar in All Three Criteria (10 families-29 individuals)

\begin{tabular}{|c|c|c|c|c|c|c|c|c|c|c|c|c|c|c|}
\hline \multirow{2}{*}{ 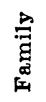 } & \multirow[b]{2}{*}{ 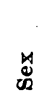 } & \multirow[b]{2}{*}{$\stackrel{\substack{\infty \\
\leftarrow}}{+\infty}$} & \multicolumn{7}{|c|}{ Free HCl at (Hours) } & \multirow{2}{*}{\multicolumn{2}{|c|}{ 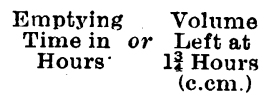 }} & \multirow{2}{*}{ Acidity } & \multirow{2}{*}{$\begin{array}{l}\text { Description of } \\
\text { Acid Curve }\end{array}$} & \multirow[b]{2}{*}{ Points of Resemblance } \\
\hline & & & $\frac{1}{4}$ & $\frac{1}{2}$ & $\frac{3}{2}$ & 1 & $1 \frac{1}{4}$ & $1 \frac{1}{2}$ & $1 \frac{3}{4}$ & & & & & \\
\hline $\mathbf{A}$ & $\begin{array}{l}\mathrm{M} \\
\mathrm{F} \\
\mathrm{F} \\
\mathrm{F} \\
\mathrm{F}\end{array}$ & $\begin{array}{l}28 \\
26 \\
2 j \\
22 \\
20\end{array}$ & $\begin{array}{l}0 \\
0 \\
0 \\
0 \\
0\end{array}$ & $\begin{array}{l}4 \\
0 \\
0 \\
2 \\
0\end{array}$ & $\begin{array}{r}8 \\
0 \\
0 \\
7 \\
11\end{array}$ & \begin{tabular}{r|}
9 \\
6 \\
0 \\
9 \\
12 \\
\end{tabular} & \begin{tabular}{r|}
11 \\
4 \\
0 \\
9 \\
13
\end{tabular} & \begin{tabular}{|r|}
7 \\
8 \\
0 \\
11 \\
13
\end{tabular} & $\begin{array}{r}2 \\
10 \\
5 \\
12 \\
13\end{array}$ & & $\begin{array}{r}80 \\
110 \\
75 \\
65 \\
15\end{array}$ & $\begin{array}{c}\text { Hypochlorhydria } \\
\text { " } \\
\text { " }\end{array}$ & $\begin{array}{c}\text { Flat } \\
\text { ": } \\
\text { " }\end{array}$ & $\begin{array}{l}\text { Stow emptying, low flat acidity } \\
\text { curves }\end{array}$ \\
\hline B & $\begin{array}{l}\text { F } \\
\text { F }\end{array}$ & $\begin{array}{l}31 \\
21 \\
29\end{array}$ & $\begin{array}{r}4 \\
13 \\
0\end{array}$ & $\begin{array}{r}8 \\
22 \\
8\end{array}$ & $\begin{array}{l}20 \\
37 \\
10\end{array}$ & $\begin{array}{l}25 \\
42 \\
23\end{array}$ & 47 & & & $\begin{array}{l}1 \\
1 \\
1 \frac{1}{4}\end{array}$ & & $\begin{array}{l}\text { Normal } \\
n\end{array}$ & 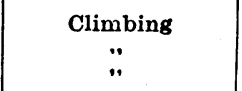 & $\begin{array}{l}\text { Rapidly emptying, sharply rising, } \\
\text { normal acidity curves }\end{array}$ \\
\hline C & $\stackrel{\mathbf{M}}{\mathbf{M}}$ & $\begin{array}{l}25 \\
22\end{array}$ & $\stackrel{0}{11}$ & $\begin{array}{r}4 \\
18\end{array}$ & \begin{tabular}{r|r}
9 \\
2.1
\end{tabular} & $\begin{array}{l}: 0 \\
24\end{array}$ & $\begin{array}{l}13 \\
25\end{array}$ & 38 & $\begin{array}{l}27 \\
42\end{array}$ & & $\begin{array}{r}5 \\
25\end{array}$ & $\begin{array}{l}\text { Normal } \\
, \bullet\end{array}$ & $\begin{array}{l}\text { Climbing } \\
" \|\end{array}$ & $\begin{array}{l}\text { Normally emntying, steadily rising, } \\
\text { normal acidity curves }\end{array}$ \\
\hline D & $\begin{array}{l}\mathrm{M} \\
\mathrm{F} \\
\mathrm{F} \\
\mathrm{M}\end{array}$ & $\begin{array}{l}60 \\
52 \\
44 \\
42\end{array}$ & $\begin{array}{l}0 \\
0 \\
0 \\
0\end{array}$ & $\begin{array}{l}0 \\
0 \\
0 \\
0\end{array}$ & $\begin{array}{l}0 \\
0 \\
0 \\
0\end{array}$ & $\begin{array}{l}0 \\
0 \\
0 \\
0\end{array}$ & $\begin{array}{l}0 \\
0\end{array}$ & & & $\begin{array}{l}1 \\
1 \frac{1}{2} \\
1 \\
1 \frac{1}{2}\end{array}$ & & 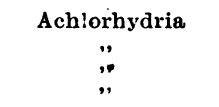 & - & Rapidly emptying; achlorhydria \\
\hline $\mathbf{E}$ & $\underset{\mathbf{F}}{\mathbf{M}}$ & $\begin{array}{l}46 \\
43\end{array}$ & $\begin{array}{l}0 \\
0\end{array}$ & $\begin{array}{l}0 \\
0\end{array}$ & $\begin{array}{l}0 \\
0\end{array}$ & $\begin{array}{l}0 \\
0\end{array}$ & $\begin{array}{l}0 \\
0\end{array}$ & $\begin{array}{l}0 \\
0\end{array}$ & & $\frac{11}{1 \frac{1}{2}}$ & & $\begin{array}{l}\text { Achlorhydria } \\
\text { " }\end{array}$ & - & $\begin{array}{l}\text { Fairly rapid emptying; achlor } \\
\text { hydria }\end{array}$ \\
\hline $\mathbf{F}$ & $\begin{array}{l}\mathbf{F} \\
\mathbf{F}\end{array}$ & $\begin{array}{l}25 \\
23 \\
21\end{array}$ & $\begin{array}{l}0 \\
0 \\
0\end{array}$ & $\begin{array}{l}0 \\
0 \\
0\end{array}$ & $\begin{array}{r}6 \\
6 \\
13\end{array}$ & $\begin{array}{r}12 \\
7\end{array}$ & & & & $1^{3}$ & & $\begin{array}{c}\text { Hypochlorhydria } \\
\ddot{"}\end{array}$ & Low rising curve & $\begin{array}{l}\text { Very rapid emptying; hypochlor- } \\
\text { hydria }\end{array}$ \\
\hline G & $\stackrel{\mathbf{M}}{\mathbf{M}}$ & $\begin{array}{l}33 \\
32\end{array}$ & $\begin{array}{l}11 \\
10\end{array}$ & $\begin{array}{l}19 \\
17\end{array}$ & $\begin{array}{l}25 \\
28\end{array}$ & 31 & $\begin{array}{l}43 \\
26\end{array}$ & $\begin{array}{l}41 \\
28\end{array}$ & & $\frac{1 \frac{1}{2}}{1 \frac{2}{2}}$ & & 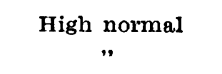 & $\begin{array}{c}\text { Falling curve } \\
\text { " }\end{array}$ & $\begin{array}{l}\text { Fairly rapid emptying, normal } \\
\text { acidity, and falling curve }\end{array}$ \\
\hline H & $\begin{array}{l}\mathbf{M} \\
\mathbf{M} \\
\mathbf{M} \\
\mathbf{M}\end{array}$ & $\begin{array}{l}24 \\
18 \\
17 \\
16\end{array}$ & $\begin{array}{l}9 \\
4 \\
0 \\
0\end{array}$ & $\begin{array}{r}14 \\
6 \\
4 \\
0\end{array}$ & $\begin{array}{r}16 \\
12 \\
4 \\
0\end{array}$ & $\begin{array}{r}14 \\
14 \\
7 \\
0\end{array}$ & $\begin{array}{r}14 \\
12 \\
4 \\
0\end{array}$ & \begin{tabular}{r|}
11 \\
24 \\
9 \\
8
\end{tabular} & 14 & 敦 & 25 & $\begin{array}{c}\text { Hypoch!orhydria } \\
\ddot{"}\end{array}$ & $\begin{array}{c}\text { Low flat curves } \\
\ddot{\#}\end{array}$ & $\begin{array}{l}\text { Normal emptying rate, low flat } \\
\text { hspochlorhydria curves }\end{array}$ \\
\hline I & $\stackrel{\mathbf{M}}{\mathbf{M}}$ & $\begin{array}{l}29 \\
25\end{array}$ & $\begin{array}{l}0 \\
0\end{array}$ & $\underset{10}{f}$ & $\begin{array}{l}26 \\
20\end{array}$ & $\begin{array}{l}44 \\
32\end{array}$ & 42 & & & l1 & & $\begin{array}{l}\text { High norinel } \\
\text { " }\end{array}$ & $\begin{array}{c}\text { Climbing curves } \\
.\end{array}$ & $\begin{array}{l}\text { Rapidly emptying, high normal } \\
\text { cimbing curves }\end{array}$ \\
\hline $\mathbf{J}$ & $\mathbf{M}$ & $\begin{array}{l}35 \\
33\end{array}$ & $\begin{array}{l}0 \\
0\end{array}$ & $\begin{array}{l}3 \\
5\end{array}$ & $\begin{array}{l}6 \\
6\end{array}$ & $\begin{array}{l}9 \\
7\end{array}$ & $\begin{array}{r}13 \\
7\end{array}$ & $\begin{array}{r}19 \\
8\end{array}$ & & $\frac{1}{1 \frac{1}{2}}$ & & $\begin{array}{c}\text { Hypochlorhydria } \\
\text { " }\end{array}$ & $\mid \begin{array}{c}\text { Low climbing to flat } \\
"\end{array}$ & $\begin{array}{l}\text { Fairly rapid emptying, fiet bypo- } \\
\text { chlorhydria curves }\end{array}$ \\
\hline
\end{tabular}


Group II.-Families in which the Test-meal Charts of all Members examined are Similar in Two of the Three Criteria, or in which the Majority of Members are Completely Similar (6 families-14 individuals)

\begin{tabular}{|c|c|c|c|c|c|c|c|c|c|c|c|c|c|c|}
\hline \multirow{2}{*}{ 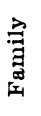 } & \multirow[b]{2}{*}{ 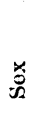 } & \multirow[b]{2}{*}{$\underset{4}{4}$} & \multicolumn{7}{|c|}{ Free HCl at (Hours) } & \multirow{2}{*}{$\begin{array}{l}\text { Emptying } \\
\text { Time in } \\
\text { Hours }\end{array}$} & \multirow{2}{*}{$\begin{array}{c}\text { Volume } \\
\text { Left at } \\
13 \text { Hours } \\
\text { (c.cm.) }\end{array}$} & \multirow{2}{*}{ Acidity } & \multirow{2}{*}{$\begin{array}{l}\text { Description of } \\
\text { Acid Curve }\end{array}$} & \multirow{2}{*}{$\begin{array}{c}\text { Points of Resemblance and } \\
\text { Difference }\end{array}$} \\
\hline & & & $\frac{1}{4}$ & $\frac{1}{2}$ & $\frac{3}{4}$ & 1 & $1 \frac{1}{4}$ & $1 ?$ & $1_{*}^{3}$ & & & & & \\
\hline $\mathbf{K}$ & $\begin{array}{l}\mathrm{F} \\
\mathrm{F} \\
\mathrm{M}\end{array}$ & $\begin{array}{l}25 \\
22 \\
17\end{array}$ & $\begin{array}{r}0 \\
13 \\
6\end{array}$ & $\begin{array}{l}10 \\
13 \\
21\end{array}$ & $\begin{array}{l}18 \\
20 \\
43\end{array}$ & $\begin{array}{l}18 \\
25 \\
52\end{array}$ & $\begin{array}{l}21 \\
27 \\
49\end{array}$ & $\begin{array}{l}36 \\
27 \\
42\end{array}$ & $\begin{array}{l}34 \\
28 \\
42\end{array}$ & & $\begin{array}{r}95 \\
110 \\
10\end{array}$ & $\begin{array}{l}\text { Normal } \\
\text { Hyperchlorhydria }\end{array}$ & $\begin{array}{l}\text { Climbing } \\
\text { Falling }\end{array}$ & $\begin{array}{l}\text { Normal to delayed emptying; } \\
\text { curves riso fairly rapidly; ono } \\
\text { hyperchlorhydria }\end{array}$ \\
\hline $\mathbf{L}$ & $\underset{\mathrm{F}}{\mathrm{M}}$ & $\begin{array}{l}50 \\
35\end{array}$ & $\begin{array}{l}0 \\
0\end{array}$ & $\begin{array}{l}0 \\
0\end{array}$ & $\begin{array}{l}0 \\
9\end{array}$ & $\begin{array}{r}0 \\
12\end{array}$ & $\begin{array}{l}0 \\
9\end{array}$ & & & $\begin{array}{l}1 \frac{1}{4} \\
1 \frac{1}{4}\end{array}$ & & $\begin{array}{l}\text { Achlorhydria } \\
\text { Hypochlorhydria }\end{array}$ & Flat & $\begin{array}{l}\text { Rapidly emptying, low acid curves; } \\
\text { achlorbydria in one }\end{array}$ \\
\hline$M$ & $\begin{array}{l}\mathbf{F} \\
\mathbf{M}\end{array}$ & $\begin{array}{l}20 \\
18\end{array}$ & $\begin{array}{l}0 \\
4\end{array}$ & $\begin{array}{l}11 \\
20\end{array}$ & $\begin{array}{l}12 \\
26\end{array}$ & $\begin{array}{l}15 \\
28\end{array}$ & $\begin{array}{l}17 \\
29\end{array}$ & $\begin{array}{l}16 \\
24\end{array}$ & $\begin{array}{l}17 \\
24\end{array}$ & . & $\begin{array}{r}10 \\
155\end{array}$ & $\begin{array}{l}\text { Normal } \\
\quad ",\end{array}$ & $\begin{array}{c}\text { Flat } \\
\text { " }\end{array}$ & $\begin{array}{l}\text { Normal acidity and shape of curve; } \\
\text { one delayed emptying }\end{array}$ \\
\hline $\mathrm{N}$ & $\underset{\mathbf{F}}{\mathbf{F}}$ & $\begin{array}{l}38 \\
35\end{array}$ & $\begin{array}{l}0 \\
0\end{array}$ & $\begin{array}{l}0 \\
0\end{array}$ & $\begin{array}{l}2 \\
2\end{array}$ & $\begin{array}{l}4 \\
3\end{array}$ & $\begin{array}{l}0 \\
4\end{array}$ & $\begin{array}{l}0 \\
2\end{array}$ & 0 & $1 \frac{1}{2}$ & 75 & $\begin{array}{c}\text { Hypochlorhydria } \\
\text { " }\end{array}$ & $\begin{array}{c}\text { Flat } \\
" \text { " }\end{array}$ & $\begin{array}{l}\text { Very low hypochlorhydria, with flat } \\
\text { curves; one delayed emptying }\end{array}$ \\
\hline $\mathbf{O}$ & $\begin{array}{l}\mathbf{M} \\
\mathbf{M}\end{array}$ & $\begin{array}{l}28 \\
21\end{array}$ & $\begin{array}{l}0 \\
0\end{array}$ & $\begin{array}{l}0 \\
8\end{array}$ & $\begin{array}{r}3 \\
12\end{array}$ & $\begin{array}{r}8 \\
12\end{array}$ & $\begin{array}{l}14 * \\
13^{*}\end{array}$ & & & $\begin{array}{l}? \\
?\end{array}$ & $?$ & $\begin{array}{c}\text { Hypochlorhydria } \\
\text { " }\end{array}$ & $\begin{array}{c}\text { Low } \\
\text { Climiving }\end{array}$ & $\begin{array}{l}\text { Hypochlorhydria, low climbing } \\
\text { curve; emptying time undeter- } \\
\text { mined }\end{array}$ \\
\hline $\mathbf{P}$ & $\begin{array}{l}\mathrm{M} \\
\mathrm{F}\end{array}$ & $\begin{array}{l}38 \\
29 \\
32\end{array}$ & $\begin{array}{l}0 \\
0 \\
0\end{array}$ & $\begin{array}{l}5 \\
3 \\
0\end{array}$ & $\begin{array}{l}6 \\
5 \\
0\end{array}$ & $\begin{array}{r}10 \\
5 \\
0\end{array}$ & \begin{tabular}{r|r}
12 & \\
6 & \\
0 &
\end{tabular} & $\begin{array}{r}14 \\
0 \\
4\end{array}$ & 19 & $\frac{1}{3}$ & 10 & $\begin{array}{l}\text { Hypochlorhydria } \\
\text { Achlorhydria }\end{array}$ & Low climbing & $\begin{array}{l}\text { mined } \\
\text { one achlorhydria }\end{array}$ \\
\hline
\end{tabular}

* Test here suspended.

Group III.-Families in which the Test-meal Charts of all Members examined are Similar in One of the Three Criteria (5 families-15 individuals)

\begin{tabular}{|c|c|c|c|c|c|c|c|c|c|c|c|c|c|c|}
\hline \multirow{2}{*}{$\stackrel{\vdots}{\overparen{\Xi}}$} & \multirow[b]{2}{*}{$\stackrel{\rtimes}{\circledast 2}$} & \multirow[b]{2}{*}{$\stackrel{9}{\leftrightarrow}$} & \multicolumn{7}{|c|}{. Frez HCl at (Hours) } & \multirow{2}{*}{$\underset{\text { Time in }}{\text { Hours }}$} & \multirow{2}{*}{ or $\begin{array}{c}\text { Volume } \\
\text { Left at } \\
13 \text { Hours } \\
\text { (c.cm.) }\end{array}$} & \multirow{2}{*}{ Acidity } & \multirow{2}{*}{$\begin{array}{l}\text { Description of } \\
\text { Acid C'urve }\end{array}$} & \multirow{2}{*}{$\begin{array}{c}\text { Points of Resemblance and } \\
\text { Difference }\end{array}$} \\
\hline & & & $\frac{1}{1}$ & $\frac{1}{3}$ & 3 & 1 & 11 & $1 \frac{1}{2}$ & $1 \frac{3}{4}$ & & & & & \\
\hline $\mathbf{Q}$ & $\begin{array}{l}\mathbf{M} \\
\mathbf{M}\end{array}$ & $\begin{array}{l}21 \\
20 \\
18\end{array}$ & $\begin{array}{l}4 \\
7 \\
0\end{array}$ & $\begin{array}{r}5 \\
15 \\
0\end{array}$ & $\begin{array}{r}16 \\
20 \\
6\end{array}$ & $\begin{array}{r}26 \\
15 \\
8\end{array}$ & $\begin{array}{r}35 \\
17 \\
0\end{array}$ & $\begin{array}{l}58 \\
19 \\
\cdot 0\end{array}$ & $\begin{array}{r}59 \\
23 \\
0\end{array}$ & & $\begin{array}{r}30 \\
130 \\
80\end{array}$ & $\begin{array}{c}\text { Hy perchlorhydria } \\
\text { Normal } \\
\text { Hypochlorhydria }\end{array}$ & $\begin{array}{c}\text { Climbing } \\
\text { Flat } \\
, "\end{array}$ & $\begin{array}{l}\text { All delayed emptying but differing } \\
\text { acidities }\end{array}$ \\
\hline $\mathbf{R}$ & $\underset{\mathrm{F}}{\mathbf{M}}$ & $\begin{array}{l}32 \\
30\end{array}$ & $\begin{array}{l}5 \\
0\end{array}$ & $\begin{array}{r}17 \\
0\end{array}$ & 20 & & & & & $\frac{3}{2}$ & & Achlorhydria & Climbing & Extremely rapid emptying \\
\hline $\mathbf{S}$ & $\begin{array}{l}\mathbf{M} \\
\mathbf{F} \\
\mathbf{F} \\
\mathbf{M}\end{array}$ & $\begin{array}{l}35 \\
30 \\
34 \\
31\end{array}$ & $\begin{array}{l}\mathbf{0} \\
\mathbf{8} \\
\mathbf{0} \\
\mathbf{0}\end{array}$ & $\begin{array}{r}0 \\
14 \\
0 \\
0\end{array}$ & $\begin{array}{r}0 \\
18 \\
0\end{array}$ & $\begin{array}{r}18 \\
18 \\
0\end{array}$ & $\begin{array}{l}18 \\
15\end{array}$ & $\begin{array}{l}20 \\
27\end{array}$ & & $\begin{array}{l}\frac{1}{2} \\
1 \\
1 \\
\frac{1}{2}\end{array}$ & & $\begin{array}{l}\text { Normal } \\
\text { Achlorhydria } \\
\text { " }\end{array}$ & $\begin{array}{c}\text { Slow climbing } \\
\ddot{-}\end{array}$ & $\begin{array}{l}\text { Two normal acidity and farly } \\
\text { quick emptying; two achylia and } \\
\text { very rapid emptying }\end{array}$ \\
\hline $\mathbf{T}$ & $\begin{array}{l}\mathbf{M} \\
\mathbf{M} \\
\mathbf{M}\end{array}$ & $\begin{array}{l}46 \\
42 \\
40\end{array}$ & $\begin{array}{r}0 \\
10 \\
0\end{array}$ & $\begin{array}{r}0 \\
28 \\
14\end{array}$ & $\begin{array}{r}0 \\
33 \\
19\end{array}$ & $\begin{array}{r}0 \\
52 \\
28\end{array}$ & 29 & & & $\begin{array}{l}1 \\
1 \\
14\end{array}$ & . & $\begin{array}{c}\text { Achlorhydriz } \\
\text { Hyperchlorhydria } \\
\text { Normal }\end{array}$ & ${ }^{\text {Climbing }}$ & $\begin{array}{l}\text { Al1 rapidly emptying, two with } \\
\text { climbing curves }\end{array}$ \\
\hline $\mathbf{U}$ & $\begin{array}{l}\mathbf{M} \\
\mathbf{F} \\
\mathbf{F}\end{array}$ & $\begin{array}{l}30 \\
32 \\
28\end{array}$ & $\begin{array}{l}0 \\
0 \\
0\end{array}$ & $\begin{array}{l}7 \\
5 \\
0\end{array}$ & $\begin{array}{r}21 \\
15 \\
0\end{array}$ & $\begin{array}{r}24 \\
18 \\
0\end{array}$ & $\begin{array}{r}30 \\
23 \\
0\end{array}$ & $\begin{array}{r}37 \\
16 \\
0\end{array}$ & $\begin{array}{r}44 \\
17 \\
0\end{array}$ & & $\begin{array}{l}40 \\
15 \\
60\end{array}$ & $\begin{array}{c}\text { Normal } \\
\text { Achlorḧhdrià }\end{array}$ & $\begin{array}{c}\text { Cl Fallinging } \\
\text { Fang }\end{array}$ & All rather delayed emptying \\
\hline
\end{tabular}
Group IV.-Families in which the Members examined show no, or few, Resemblances in their Test-meal Charts
(6 families-20 individuals)

Six family groups, composed of $5,4,3,3,3$, and 2 members respectively.

Group V.-Parents and Children (4 families-8 individuals)

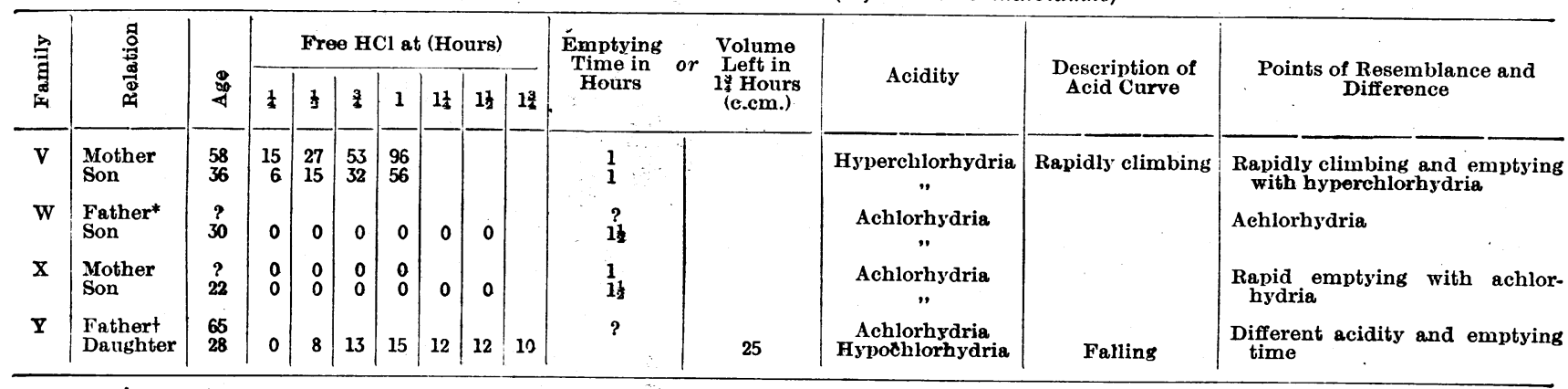

* According to the son, who is a medical man, the fither died of pernicious anaemia, a conditio 1 invariably, or ahnost invariably,

t The father, a patient of one of as (F. L. A.), aied of pernicicus anaemia.

In some of the other groups, although this complete resemblance did not extend to all members, there were nevertheless pairs in a few of the larger families which were similar in all, or in nearly all, respects. Occasionally, where two distinct types appear in a family, possibly some resemble one parent and some the other. Although the above evidence strongly supports Hurst's contention that gastric types run in families (gastric diathesis) obviously the matter could be proved only by examining all members of several families over two or three generations while each member was still comparatively young and before gastritic or senile changes had occurred. Gastric function might prove to be hereditary in a Mendelian sense. To carry out such a set of observations would be almost impossible, owing to the difficulty of finding and persuading all members to undergo the tests, though until this is done we are hardly justified in using the term "familial" and in drawing other than tentative con- 
clusions from our observations. A careful examination of our figures; however, would make it appear probable that such a relationship does exist.

\section{Discussion}

That gastric acidity, the form of the acidity curve, and the emptying time of the stomach should be similar in the various members of a family would not be surprising in view of previous observations. Apperly and Semmens ${ }^{6}$ have shown that these characters depend largely on the tonus and activity of the gastric musculature and on certain chemical constituents of the blood. Further, the relationship of these characteristics to the ratio of pulmonary surface to body weight, and therefore ultimately to the build of the body and its constitution generally, lends further support to the hypothesis that they express family characteristics.

The marked resemblance in the test-meal charts in a large number of cases is, however, from another point of view rather unexpected. When we remember that in all instances the subjects were experiencing a test-meal experiment for the first time, with all grades of psychic disturbances consequential to the examination, the results seem to show that such influences count for much less than we have been taught to believe, unless we assume that all members of each family reacted psychically in the same manner. In actual fact this was not so, marked differences in psychic reaction being noted among the several members of the same family. It has indeed long been our view that temporary psychic disturbances have little appreciable effect on either the general form and magnitude of the acidity curve or on the emptying time of the stomach.

\section{Conclusions}

It has been asserted by various writers that the several varieties of normal gastric function are often inherited characteristics. We have investigated this question in a series of 31 families comprising 86 individuals, and our results support these statements.

\section{REFERENCES}

1 Hurst: Lancet, 1922, ii, 1369 ; Ibid., 1923, i, 111

2 Apperly: Supplement to Med.. Journ. of Australia (Transactions of Congress), March, 1924, 83

3 Bennett and Ryle: Guy's Hospital Reports, 1921, 1xxi, 286.

- Baird, Campbell, and Hern: Ibid., 1924, lxxiv, 339.

- Apperly and Semmens: Med. Journ. of Australia, August, 1928, 237.

- Idem: Ibid., August, 1928, 226.

Idem: Ibid., August, 1929, 193.

\section{THE INCISION FOR APPENDICECTOMY}

BY

A. H. SOUTHAM, M.D., M.Ch.Oxon. F.R.C.S.ENG.

HONORARY ASSISTANT SURGEON, MANCHESTER ROYAL INFIRMARY ; LECTURER IN OYERATIVE SURGERY, MANCHESTER UNIVERSITY

Injury to a nerve is always an accident of some gravity, and injuries to the nerves of the abdominal wall are more common than is perhaps generally recognized. As a resuit of such injuries, atrophy of muscle is apt to follow, leading to some functional interference and incapacity.

The problem which is the best incision for the removal of the acutely inflamed appendix is constantly exercising the minds of surgeons. In a paper ${ }^{1}$ published in 1924, in which an account was given of the after-results of the different types of abdominal incisions in general use, it was pointed out that a right inguinal hernia sometimes followed the McBurney muscle-splitting incision after operation in cases of acute appendicitis. Since that date several more cases showing this complication have been seen, enabling further observations to be made on this subject.
Incidence

It is difficult to give any accurate estimate of the frequency of the appearance of a right inguinal hernia following the use of the gridiron incision, as cases of this nature are seldom recorded, and it is doubtful if the connexion between the appendix operation and the subsequent appearance of the hernia is often recognized. The following figures are of some interest.

Roberts $^{2}$ states that the City of London Truss Society meets with twelve to twenty cases of this kind a year following the gridiron incision, and that he has never seen a hernia which occurred after the rectus incision. Griffiths ${ }^{3}$ records ten examples in 100 consecutive cases of hernia, nine following the McBurney incision and one the Battle incision. In the latter case there was a large sac of the infantile type, the patient being probably predisposed to hernial formation. In describing the McBurney incision Romanis and Mitchiner ${ }^{4}$ state that care must be taken not to damage the ilio-inguinal nerve, as this results in post-operative inguinal hernia-a complication seen in some 30 per cent. of cases. The hernia may appear at any time from a few months to a few years after operation; six months is about the usual period.

\section{Etiology}

In the paper already referred to it was pointed out that the hernia probably resulted from some injury inflicted on the ilio-inguinal nerve during the operation for the removal of the appendix.

It will be recalled that the ilio-inguinal and ilio-hypogastric nerves are branches of the lumbar plexus, and supply the muscles of the flank. The former runs forward on the iliacus and pierces the transversalis abdominis muscle near the anterior part of the iliac crest, and lies between the transversalis and internal oblique muscles. A short distance in front of the anterior superior iliac spine it passes through the internal oblique and then, descending in the inguinal canal, becomes superficial, after emerging through the external abdominal ring to supply the skin over Scarpa's triangle and the root of the penis. During its course the ilio-inguinal nerve gives muscular branches to the muscles below the anterior superior iliac spine. It is at the point where the nerve lies internal to the anterior superior iliac spine that it is liable to be damaged. This may take place when the muscle fibres of the internal oblique and transversalis are being split in making the incision, or the nerve may be included in the sutures when closing the wound, or, again, it may be involved in scar tissue at a later date, particularly in cases of abscess where drainage has been necessary. The nerve lesion results in paralysis of the muscles and the conjoined tendon round the internal abdominal ring and in the subsequent appearance of an inguinal hernia.

In three recent cases of right inguinal hernia following the muscle-splitting incision, where a second operation was undertaken for the radical cure of the hernia, the opportunity was taken to excise a portion of the ilioinguinal nerve as it lay in the inguinal canal. (Un removal the three nerves were kindly examined by Professor J. S. B. Stopford, who reports as follows:

All three nerves showed partial degeneration. The proportion of degenerate fibres varied in the three specimens; in two about half the fibres were affected, whilst in the other only about one-third. There was no evidence of interstitial changes, and the histological findings were consistent with those succeeding compression of a nerve by such things as scar tissue.

\section{Comment}

The choice of incision in a case of acute appendicitis merits the serious consideration of every surgeon. The view here taken is that in the McBurney muscle-splitting 